\title{
Pose Invariant Palmprint Recognition
}

\author{
Chhaya Methani and Anoop M. Namboodiri \\ International Institute of Information Technology, Hyderabad, India \\ chhaya@research.iit.ac.in, anoop@iit.ac.in
}

\begin{abstract}
A palmprint based authentication system that can work with a multipurpose camera in uncontrolled circumstances, such as those mounted on a laptop, mobile device or those for surveillance, can dramatically increase the applicability of such a system. However, the performance of existing techniques for palmprint authentication fall considerably, when the camera is not aligned with the surface of the palm. The problems arise primarily due to variations in appearance introduced due to varying pose, but is compounded by specularity of the skin and blur due to motion and focus. In this paper, we propose a method to deal with variations in pose in unconstrained palmprint imaging. The method can robustly estimate and correct variations in pose, and compute a similarity measure between the corrected test image and a reference image. Experimental results on a set of 100 user's palms captured at varying poses show a reduction in Equal Error Eate from $22.4 \%$ to $8.7 \%$.
\end{abstract}

\section{Introduction}

The use of digital imaging, specifically camera based imaging, for biometric authentication has changed the applicability of biometric authentication for personal security. Camera based imaging is fast, inexpensive, convenient to use, and is easily available to the common man. In addition to biometric modalities such as face, gait, etc., one can employ camera based imaging for fingerprints, palmprints, or even handwriting. It also facilitates the use of multi-modal biometrics, which alleviates many concerns on accuracy and applicability, as it is possible to capture different biometric features using the same imaging setup and at the required resolution.

Despite all the advantages, there are many challenges to overcome to realize biometric authentication systems that use unconstrained camera-based imaging. The major problems include background clutter or noise that make segmentation difficult, illumination changes that can affect the feature extraction process, scale and resolution changes, and the increased flexibility offered to the subject in terms of pose changes. The onus then lies on the algorithm to accommodate all these variations in the best possible way. This adversely affects the recognition rate that is much higher when limited degree of freedom is allowed. As a result, many camera based recognition systems employ a capturing setup that constrains the pose and scale, and provides uniform illumination and constant background. Such a setup may not add much in terms of cost, but makes the apparatus cumbersome to use, liable to frequent damage, less intuitive and user friendly, and very specific to a particular biometric. Hence, there is a potential to develop new algorithms that are more robust to environmental variations and also 


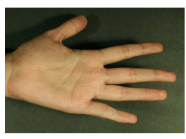

(a)

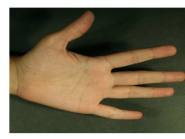

(b)

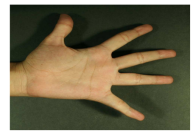

(c)

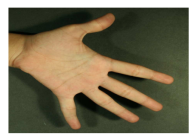

(d)

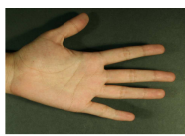

(e)

Fig. 1. Variations in hand pose due to unconstrained imaging

allow more flexibility to the subject. Efforts in this direction include achieving pose and illumination invariance for face recognition [1], view invariance for gait recognition [2] and handling different poses for hand geometry based authentication [3].

In this paper, we look at the problem of achieving pose invariance in palmprint based authentication. Initial palmprint matching systems used scanned images from inked palms. A detailed image having the major lines, wrinkles and even creases of the palm was obtained. Approaches developed for these images involved matching datum points, and the line orientation at them [4]. This constituted offline palmprint matching. RealTime Online Palmprint matching was made possible with the use of electronic imaging by capturing low resolution images using devises such as web cameras. The assumptions of controlled environment and restricted palm pose were convenient, and only prominent palm lines and their structure were used for matching [5].

As both hand-geometry and palmprint use the same imaging setup, one could also combine them both in a multi-biometric setup [6]. The common preprocessing step computes the two valley points $p_{1}$ and $p_{2}$ as shown in Figure 2 . As the palm is cut out with respect to the coordinate axes formed by joining these two stable points on the palm, the extraction process becomes invariant to in-plane rotations and translation. Focus of current research is, hence, to find better ways for representation of palm [7] and discovering new ways of feature representation for improved and robust matching. Popular representations include Gabor filter responses [5], wavelet basis representation, Fourier representation, orientation histogram based etc. [7],.

The partial rotation and translation invariance to in-plane rotation and translation is achieved by constrained pose hand imaging. This suffers from problems relating to user inconvenience, being less intuitive, unsatisfactory user cooperation, a cumbersome setup and limiting the possibilities of extending the system. These issues must be addressed to make the system more robust and better adept to practical scenarios. Approaches to deal with varying poses either try to compute pose-invariant features, or apply a transformation to the image to normalize pose variations.

G. Zheng et al in [3] proposed projective invariant hand geometry features. In [8], Doublet et al. propose novel way of contact less hand recognition. The approach primarily concentrates on extracting the palm from an image with cluttered background. Their hand detection method is based on a combination of skin color modeling and shape models. For the purpose of recognition, the approach assumes that the palm is being held parallel to the camera's imaging plane.

In this work, we extend their approach to include matching images with hand variations in 3D space(see Figure 1). We deal with projective variations that will make palmprint recognition possible using a truly unconstrained imaging setup. Pose variations distort the absolute position and relative distance between the lines, and change 


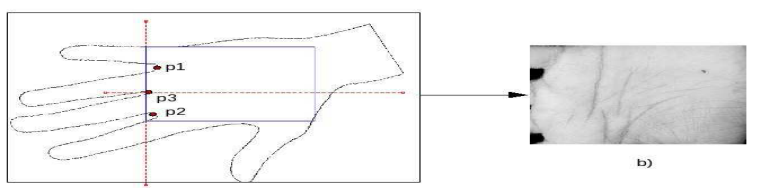

Fig. 2. Extraction of palm region based on finger valleys

their characteristic properties like length, width, curvature, aspect ratio etc. Hence, traditional matching can not be applied directly to images having different poses.

In this paper, we approach the problem of unconstrained palmprint imaging and the related pose variations for the first time. We propose a novel algorithm for pose invariant palmprint matching. As a part of the algorithm, we propose a robust image alignment technique and a method to detect the orientation of the hand. We show the results of our approach on a synthetic and a real dataset. The synthetic dataset gives us an opportunity to isolate, quantify and incrementally study the effects of pose changes, while the real dataset accurately models the practical problems such as skin deformations, specular reflections, etc. Some images from our dataset are shown in Figure 5.

The rest of the paper is organized as follows. Section 2 explains the approach chosen to attain pose invariance and reasons for it. Section 3 describes the practical challenges in image alignment and the solution. Section 4 gives a step wise formulation of the solution proposed by us, followed by experimental results in Section 5 .

\section{Unconstrained Palmprint Imaging}

In our work, we consider images taken with unrestricted and mostly unsupervised hand motion. This may lead to a change in hand pose. Typical set of images to be matched is shown in Figure1(a) and1(b). Matching can be done in two ways.

Finding Projective Invariant Features in Palm. As pointed out in [3], when subjected to geometrical projective transformations, cross ratios created by 5 coplanar points with a specific configuration are invariant to pose change. The approach assumes the planarity of hand, and the landmark points are chosen from the the finger creases.

The bottleneck of this is that it requires us to find 5 stable landmarks with specific geometric relationship on the palm images. However, considering the structure of palm, which consists of three major lines: the heart, head and life lines, the number of distinctive points that can be reliably identified are very limited. Points formed by intersection of two lines are not reliably detected due to blur, illumination and resolution changes, and the problem increases further due to introduction of wrinkles during pose changes. In short, the palm images are weakly textured and do not have enough acceptable landmark points to consider the computation of projective invariant features.

Pose Correction. A second approach is that of explicitly determining the pose and then correcting for it. A fundamental matrix generally models the transformation between objects in $3 \mathrm{D}$ world $\left(\left[x^{\prime}, y^{\prime}, z^{\prime}\right] \mathcal{F}[x, y, z]^{T}=0\right)$. However, we can restrict the 
set of possible transformations, by assuming the thickness of the palm to be negligible, approximating it to a planar surface. It should be noted here that the palm, when in different poses, becomes prone to deformations of the skin because of the movement of the fingers and the thumb. Hence, additional line like impressions are introduced. However, these deformations can be ignored if the palm is relatively flat, and for our current purpose, we choose to ignore the skin deformations. If we assume the palm to be a rigid plane, correction of pose variation becomes well posed. From multi-view geometry of imaging, two images of a planar object are always related by a linear transform, characterized by 8 parameters [9]. Given any point in one image, in the homogeneous coordinate system, $[x, y, 1]^{T}$, the corresponding point in the second image is given by:

$$
\left[x^{\prime}, y^{\prime}, c\right]=\mathcal{H}[x, y, 1]^{T},
$$

where the actual image points are $x^{\prime} / c$ and $y^{\prime} / c$. The Homography matrix, $\mathcal{H}$, can be estimated from correspondences between two views, up to scale. Hence $\mathcal{H}$ has 8 unknowns and we need 4 point correspondences to solve it. But, as mentioned earlier, it is difficult to find robust correspondence points in the weakly textured palm image. Although the flexibility offered by RANSAC in choosing the initial point set, makes it possible. We call this correction the Image Alignment stage, which is described below.

\section{Image Alignment}

Our goal is to align two palm images with each other so as to match them properly. For this, we need to find 4 point correspondences for applying Homography for pose correction. In the absence of robust correspondences, we use a set of interest points as the initial correspondence and try to improve it using RANSAC. The two most stable points on the palm are the valley points $p_{1}$ and $p_{2}$ shown in Figure 2, and we directly know the correspondence between them. For the other two correspondences, we search the palm lines for any points with a strong surrounding context in both images.

The process of interest point detection proceeds as follows: We find an edge map of the lines using a curvature based method proposed in [10]. The approach is more robust to noise, and extracts prominent lines in the image. However, in many cases the edge map is still noisy. So, we adopt a two-step line detection algorithm. First, two edge images are computed with a high and a low threshold(Figure 3 (b) and (c)). The image with higher threshold has parts of all the major lines and prominent wrinkles. We use these points as seeds to search in the low-threshold image using morphological operations to get the final edge map. The final image obtained is shown in Figure 3(d).

In the second step, we richly sample points from edge map as indicated by the cross marks in Figure 3(e). These points form the initial candidates that have the highest probability of being the interest points. The initial point set is culled by removing those without significant correspondence in the second image. The correspondence level is estimated by using correlation(See Algorithm 1 . The list of corresponding points selected by this process is referred to as putative matches.

There are possible scenarios where the correspondences found are incorrect, since we have used the local context only. Another possibility is the introduction of additional interest points due to skin deformation or illumination difference. To compute a reliable 


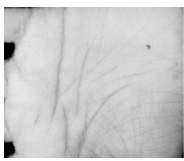

(a)

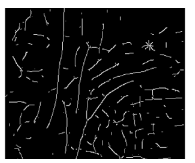

(b)

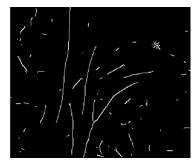

(c)

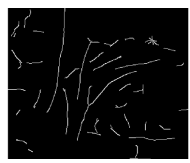

(d)

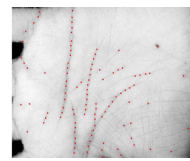

(e)

Fig. 3. Steps being followed to get interest points from the image. a) Palm Image, b) Edge map with a lower threshold, c) Edge map with a higher threshold, d) Edge map after enhancing image in c, e) Sampled interest points marked on the palm image.

estimate of homography, we use a RANSAC based method [11]. The input to RANSAC is $n$ pairs of putative matches, including the 2 points computed from the finger valleys. We modify the traditional RANSAC algorithm slightly to always include the two valley points in the set of 4 points, as we have high confidence in them.

Finally, we transform the test image with the computed Homography matrix. This results in the sample image being modified as per the dimensions of the template. Homography computation is sensitive to even small errors in point correspondences. The efficacy of the entire algorithm finally depends on how good correspondences turn out to be. The overall process of image alignment is presented in Algorithm 1 . A typical set of input images and the aligned output image are shown Figure 4

\section{Proposed Solution}

The overall matching algorithm includes preprocessing, extraction of the region of interest (ROI), image alignment, feature extraction, and matching.

Preprocessing. Consists of three steps, a)Extracting hand from the background and b)Determining the orientation of the hand, and c)locating the two finger valleys.

To segment out the hand from a cluttered background, an ada boosting based approach was proposed by Doublet et al. [8]. We use a uniform background in our experiments as our focus is to solve the problem of unknown hand pose for matching. Next, we need to estimate the approximate orientation of the palm. We do this by computing the direction of highest variation among the foreground pixels(largest Eigen vector). If $I$ is the palm image with dimensions $m \times n$, then we take a matrix $M=[X Y]$, where

$$
X=x_{i}, Y=y_{i} \mid 1 \leq x_{i} \leq m, 1 \leq y_{i} \leq n \& I\left[x_{i}, y_{i}\right] \neq 0
$$

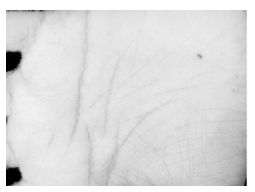

(a)

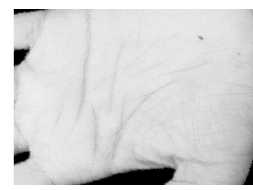

(b)

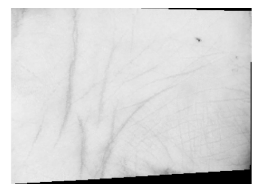

(c)

Fig. 4. Result of aligning Image a to Image b, using Homography, has been shown in Figure c 


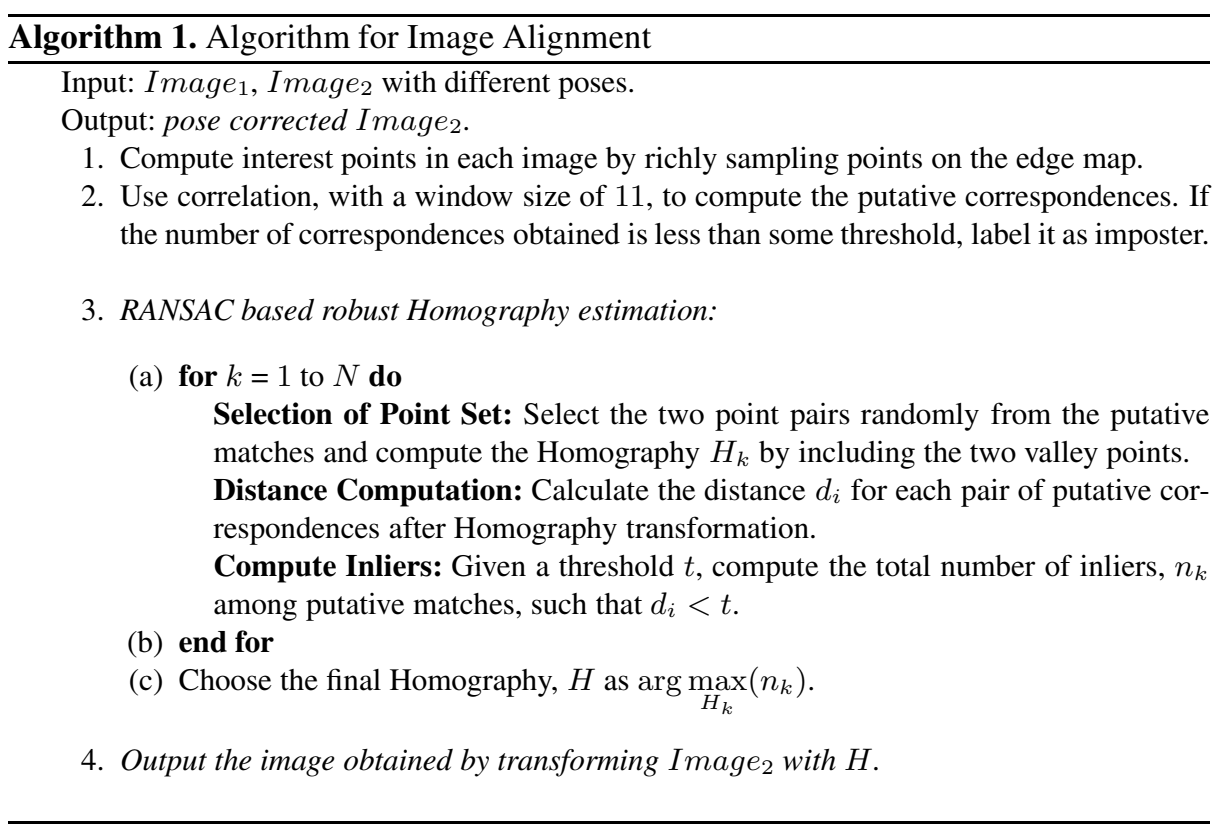

The largest eigen value of the scatter matrix, $S$, of $\mathrm{M}$ above gives us the orientation of the palm. This method corrects large degrees of in-plane rotations as opposed to previous approaches. Finally, we extract the valley points as in [5].

Extraction of ROI. The region of interest in the hand image is defined with respect to the two valley points detected above. We assume the middle valley point, $p_{3}$ to be the origin, and compute the line passing through $p_{3}$ and parallel to the line joining $p_{1}$ and $p_{2}$ (Figure 2). We then extract the maximal rectangular region aligned to the above line, containing forerground pixels. This allows us to consistently extract the entire visible area of the palm, irrespective of the change in view. Note that this is an approximate region of interest, and the matching is done after image alignment.

The palm extraction process indirectly solves the problem of variations in palm size. In approaches assuming a fixed palm to camera distance, palm area of fixed size is cut out from all the subjects. In such a case, the subjects with palm size smaller than the fixed size are either rejected or are able to infiltrate the system. This problem was raised by Poon et al. [12], who proposed the use of variable sized elliptical ROI to address it. By extracting variable sized palm areas, we are able to handle this problem as well.

Image Alignment. Image alignment algorithm was described in Section 3. In most cases, we get two images of the same size that are suitably aligned to each other. However, in certain cases, it fails to compute an accurate match. RANSAC is a robust algorithm for Homography computation, and any failure to compute a valid transformation indicates errors in point correspondence. The reason is lack of correlation at local windows of points. So, we assume that palm textures are different and assign a high dissimilarity value, effectively labeling them as belonging to different individuals. 
Feature Extraction. Once the images are aligned, one can use any one of the existing approaches for matching palmprints. In our experiments, we match responses of Gabor Filters from corresponding windows and their neighborhood, as proposed in [5].

Matching. A typical pose corrected image is shown in Figure 4 (c). To match the images, we only concentrate on the overlapping parts of the two palms. This is referred to as matching with masking, and the distance is computed as the sum of hamming distances between the binarized real and imaginary filter responses [5]. For certain pairs of genuine matches, error in image alignment can reduce the resulting matching score. To overcome this, we define the final dissimilarity score as $d_{\text {final }}=\min \left(d_{\text {fixed }}, d_{\text {corrected }}\right)$, where the individual dissimilarity scores are computed assuming fixed pose, and after pose correction. If Homography computation is accurate, the dissimilarity score after pose alignment should reduce for genuine pairs.

In short, we compute the best available matching score between any two image pairs. This reduces the variability in genuine matching scores. However, it also improves the matching scores of imposter pairs. The result of this bias towards better match is clearly visible in the results presented in the following section.

\section{Experimental Results and Discussion}

We present results of the proposed approach on PolyU dataset with synthetically generated pose variations along with a real dataset with varying palm poses.

Synthetic Dataset. We selected 50 individuals from the PolyU database, and generated various poses. For each subject, we randomly pick 1 image, apply out of plane rotations, and get images from the resulting view. We generated 9 different poses for each subject by rotating the image about $\mathrm{X}$ and $\mathrm{Y}$ axes ( $\mathrm{Z}$ being the camera axis), with angles varying from $0^{\circ}$ to $45^{\circ}$. Figure 5 shows some of the examples from two classes.

To compare our approach with the traditional approach, which assumes a fixed pose (except in-plane rotation and translation), we obtained two values of matching score between every pair, with and without image alignment. Note that the fixed pose approach always extracts a fixed-sized square ROI. Each image was matched with 4 registered
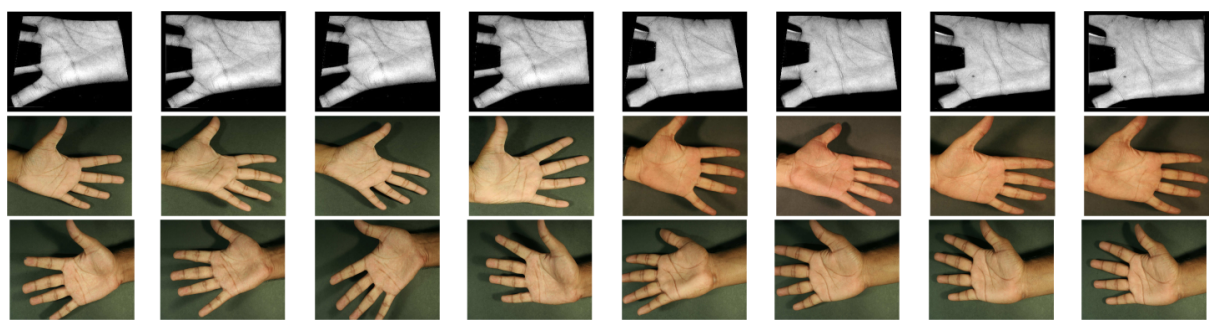

Fig. 5. A set of images from the Dataset. First row shows 4 images each from 2 users in Synthetic Dataset. 4 left hand images each from 2 users in Real Dataset are shown in the second row and right hand is shown in third row. 
images, and the minimum score is taken as the matching score. Comparison of ROC curves between our algorithm and fixed pose approach, for Synthetic Dataset, is given in Figure 6(a). A semilog plot of the same is given in figure 6(c), to highlight the low FAR regions.

Real Dataset. The synthetic dataset does not model the complexities of unconstrained real world imaging. In reality, variations arising from illumination changes, skin deformation, concave nature of the palm, and specular reflection from the skin, should be considered to check the effectiveness of a matching algorithm(Figure 7). We thus created a real world dataset. The setup consists of a fixed camera looking at a uniform background. We collect 5 different palm images with varying poses from each subject. We consider the right and left palms of a user as two different classes, and the dataset consists of 100 classes coming from 50 individuals. However, for the purpose of computation of imposter distances, we do not compare an image from a right palm to a left palm. Figure 5 shows four examples each from four classes from the dataset.

For every user, 2 images were used as the registration images. And the rest were used for testing. Two matching scores for each pair were computed using the fixed pose and the proposed approaches. The ROC curves for the two have been presented in Figure 6.b) and (d).

Table 1 compares the Equal Error Rate while using the two approaches. For synthetic data, we categorized pose variations in 5 groups to analyze performance incrementally. We also present the results while blindly applying the pose correction using Homography along with the complete matching algorithm. From the results on synthetic data, we note that the pose correction step is inaccurate and introduces additional errors in case of data with small pose variations. However, as the extent of pose variation increases, the correction phase in the proposed approach becomes beneficial, increasing the overall accuracy of the matcher.

Table 1. Equal Error Rate(EER) on two datasets with the fixed pose and proposed approach

\begin{tabular}{|c|l|l|l|l|l|l|}
\hline Method & \multicolumn{5}{|c|}{ Synthetic Data } & Real Data \\
\hline & $0^{\circ}-20^{\circ}$ & $20^{\circ}-30^{\circ}$ & $30^{\circ}-35^{\circ}$ & $35^{\circ}-40^{\circ}$ & $40^{\circ}-45^{\circ}$ & \\
\hline Fixed Pose Approach & $0.01 \%$ & $3.24 \%$ & $3.71 \%$ & $16.93 \%$ & $30.92 \%$ & $22.4 \%$ \\
\hline Blind Pose Correction & $16.48 \%$ & $12.40 \%$ & $11.14 \%$ & $14.98 \%$ & $11.92 \%$ & $16.51 \%$ \\
\hline Proposed Approach & $0.47 \%$ & $4.19 \%$ & $11.14 \%$ & $14.98 \%$ & $11.92 \%$ & $8.71 \%$ \\
\hline
\end{tabular}

A few interesting characteristics to be noted in ROC plot of real data in Figure 6(d):

- $(p)$ :Note that the GAR drops even with high FAR scores. This indicates the presence of some genuine pairs with low similarity. This can arise in uncontrolled imaging scenarios from variations due to blur, wrinkles, illumination, etc.(Figure 7)

- $(q)$ : The second region of interest is to the left with low GAR, which indicates the presence of image pairs from different palms with very high similarity. This arises mainly due to presence of images with most of the pixels being saturated due to specular reflections from the skin(Figure7(c)). 


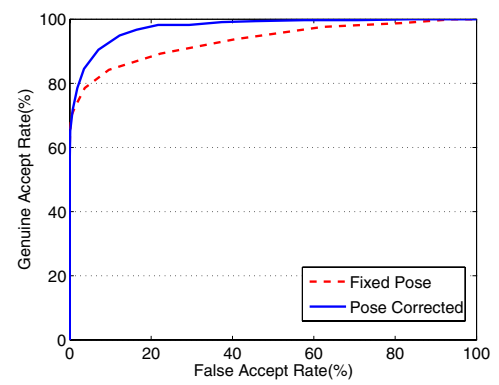

(a) Synthetic Data

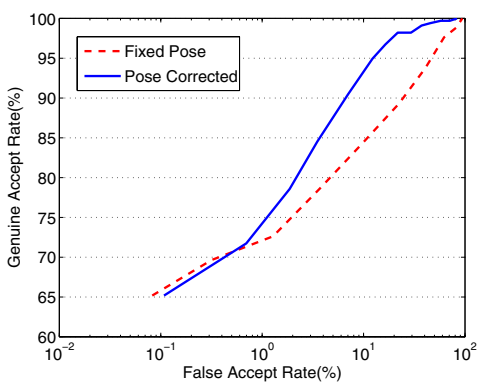

(c) Synthetic Data (semilog)

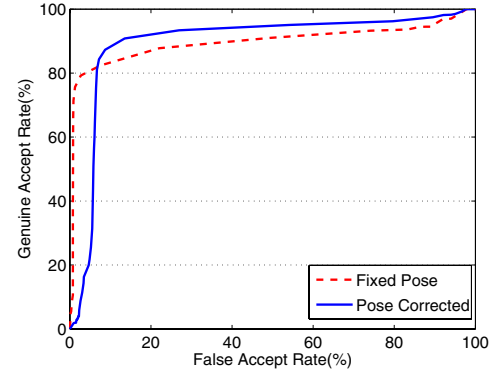

(b) Real Data

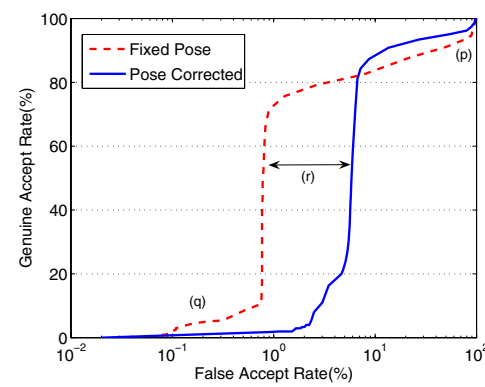

(d) Real Data (semilog)

Fig. 6. ROC curves for the Synthetic and Real Datasets in linear and logarithmic scales

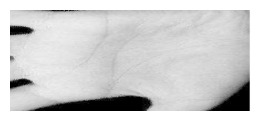

(a)

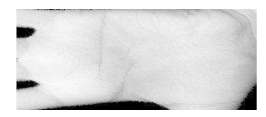

(b)

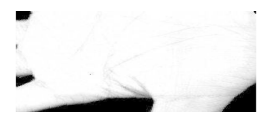

(c)

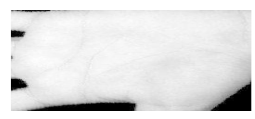

(d)

Fig. 7. Images from our dataset with blur, specular reflection, and skin deformations

- $(r)$ : Note the drop in GAR in pose corrected version is earlier than the uncorrected one.The reason being that pose correction reduces distance between pairs from different palms too, making some of them closer to genuine pair distance.

One can rectify some of the above problems by improving the capturing device. For example, to reduce the effects of specular highlights(p), use a polarizing filter and improve the gain control of the camera. Improvements in camera sensor and lens can also lead to a reduction in blur(q). The last problem is inherent to the approach of pose correction, and requires better matching algorithm to overcome.

\section{Conclusion and Future Work}

In this paper, we presented a novel approach for enabling unconstrained palmprint recognition over a variety of poses. The dataset we collected contains significant amount 
of variations due to pose, illumination and specularity. We describe the challenges faced by such a system and proposed a mechanism to address the pose changes. We found that recognition accuracy is better for well-focused images as it is easier to find stable corresponding points across poses. The accuracy of the system is mostly affected by the imposter matches turning good because of a flexible alignment process. Our method achieves considerable reduction in the EER from $22.4 \%$ to $8.7 \%$ for a set of 100 users.

One could imagine the use of additional information such as skin color, hand shape, and positions of finger creases, etc. to improve the overall accuracy of matching. One could also improve the accuracy of the approach using better imaging, and using more accurate methods for correspondence computation. One could also employ skin deformation models or using articulated or concave models for the palm surface.

\section{References}

1. Kahraman, F., Kurt, B., Gokmen, M.: Robust face alignment for illumination and pose invariant face recognition. CVPR (November 2007)

2. Kale, A., Chowdhury, A.R.: Towards a view invariant gait recognition algorithm. In: IEEE Conference on AVSS, pp. 143-150 (2003)

3. Zheng, G., Wang, C.J., Boult, T.E.: Application of projective invariants in hand geometry biometrics. IEEE Transactions on Information Forensics and Security 2(4), 758-768 (2007)

4. Duta, N., Jain, A.K., Mardia, K.V.: Matching of palmprints. Pattern Recognition Letters 23, 477-485 (2002)

5. Zhang, D., Kong, W.K., You, J., Wong, M.: Online palmprint identification. IEEE Trans. Pattern Anal. Mach. Intell. 25(9), 1041-1050 (2003)

6. Kumar, A., Wong, D.C.M., Shen, H.C., Jain, A.K.: Personal verification using palmprint and hand geometry biometric. In: Kittler, J., Nixon, M.S. (eds.) AVBPA 2003. LNCS, vol. 2688, pp. 668-678. Springer, Heidelberg (2003)

7. Sun, Z., Tan, T., Wang, Y., Li, S.Z.: Ordinal palmprint representation for personal identification. In: Proc. IEEE Computer Vision and Pattern Recognition (CVPR), pp. 279-284 (2005)

8. Doublet, J., Lepetit, O., Revenu, M.: Contact less hand recognition using shape and texture features. ICSP Proceedings 3 (2006)

9. Hartley, R., Zisserman, A.: Multiple view geometry in computer vision (2000)

10. Garg, S., Sivaswamy, J., Chandra, S.: Unsupervised curvature-based retinal vessel segmentation. In: Proc. of IEEE International Symposium on Bio-Medical Imaging(ISBI), pp. 344 347 (2007)

11. Xiaowei, L., Yue, L., Yongtian, W., Dayuan, Y.: Computing homography with ransac algorithm: a novel method of registration. In: Proceedings of the SPIE, vol. 5637, pp. 109-112 (2005)

12. Poon, C., Wong, D., Shen, H.: A new method in locating and segmenting palmprint into region-of-interest. In: Proc. of 17th International Conference on Pattern Recognition (ICPR 2004), pp. 1051-1054 (2004) 